\title{
El uso de la wiki y la mejora en el aprendizaje colaborativo
}

\section{The Use of the Wiki and the Improvement in Collaborative Learning}

\author{
Juan José Flores-Cueto* (D) \\ Universidad de San Martin de Porres, Lima, Perú \\ ORCID: https://orcid.org/0000-0003-3251-4463 \\ Rafael Garay Argandoña \\ Universidad de San Martin de Porres, Lima, Perú \\ ORCID: https://orcid.org/0000-0003-2156-2291 \\ Ronald M. Hernández \\ Universidad San Ignacio de Loyola, Lima, Perú \\ ORCID: https://orcid.org/0000-0003-1263-2454
}

Recibido 18-08-19 Revisado 15-10-19 Aprobado 15-12-19 En línea 31-01-20

*Correspondencia

Email: jjflorescueto@gmail.com
Citar como:

Flores-Cueto, J., Garay-Argandoña. R., \& Hernández, R. M. (2020). El uso de la wiki y la mejora en el aprendizaje colaborativo. Propósitos y Representaciones, 8(1), e447. doi: http://dx.doi.org/10.20511/pyr2020.v8n1.447 


\section{Resumen}

El avance de la tecnología ha permitido la creación de entornos de aprendizaje que favorecen la interacción social, logrando identificar aspectos claves para el desarrollo de actividades colaborativas, con relación a la conformación de grupos. El presente estudio tiene por objetivo determinar en qué medida la utilización de la wiki, en el contexto de enseñanza mediante un aula virtual, permite mejorar la colaboración en el desarrollo de trabajos grupales de estudiantes de una universidad privada de Lima. Se emplea como método de investigación el diseño cuasi experimental, con pretest y postest, un grupo intacto y un grupo experimental. El instrumento utilizado constituye una rúbrica que evalúa las dimensiones del aprendizaje colaborativo: interdependencia positiva, construcción de significado y relaciones psicosociales. Los resultados mostraron que el uso de la wiki mejora el aprendizaje colaborativo en los estudiantes de pregrado.

Palabras claves: Tecnología educacional; Aprendizaje colaborativo; Aprendizaje en línea; Estudiante universitario.

\section{Summary}

The advancement of technology has allowed the creation of learning environments that favor social interaction, identifying key aspects for the development of collaborative activities, in relation to the formation of groups. The purpose of this study is to determine to what extent the use of the wiki, in the context of teaching through a virtual classroom, allows to improve collaboration in the development of group work of students from a private university in Lima. The quasi-experimental design, with pretest and posttest, an intact group and an experimental group is used as a research method. The instrument used constitutes a rubric that evaluates the dimensions of collaborative learning: positive interdependence, meaning construction and psychosocial relationships. The results showed that the use of the wiki improves collaborative learning in undergraduate students.

Keywords: Educational Technology; Collaborative Learning; Online Learning; University Student.

\section{Introducción}

Las tecnologías de información y comunicación (TIC) desempeñan un rol fundamental en la configuración de la sociedad y cultura (Adell, 2006; Marqués, 2013; Hernández, 2017) este cambio en la sociedad es tan veloz que los sistemas de formación antiguos no pueden dar respuesta inmediata a las necesidades educativas actuales y futuras (Gairin \& Martin, 2004; Abramson, 2011; Gómez Francisco, \& Rubio González, 2017). Esto ha permitido la aparición de nuevos escenarios educativos, donde las nuevas tecnologías no solo se van incorporando a la necesidad de aprender, sino que son utilizados como medios de comunicación al servicio de la formación, como medios en los cuales tendrá lugar el proceso de enseñaza-aprendizaje (Adell, 1997; Lozano Martínez; Ballesta Pagán; Alcaraz García; Cerezo Máiquez; 2013; Córdoba Castrillón, López Murillo, Ospina Moreno, \& Polo, 2017).

En este contexto, la Web 2.0 multiplica las posibilidades de aprender al compartir contenidos, experiencias y conocimientos. El principal valor que ofrecen las aplicaciones Web 2.0 es la simplificación de la lectura y escritura en línea de los estudiantes. Esto se traduce en dos acciones sustantivas del proceso de aprendizaje: generar contenidos y compartirlos (Cobo y Pardo, 2007; Boza Carreño, Conde Vélez, 2015; Rodrigo Cano, Aguaded Gómez \& García Moro, 2019). 
En tal sentido, se puede considerar la posibilidad de utilizar aplicaciones Web 2.0 para el desarrollo de proyectos colaborativos on-line que faciliten la colaboración y participación entre estudiantes.

La experiencia docente en el aula, tanto a nivel de los procesos que se generan dentro de ella como en los resultados del aprendizaje que evalúa un docente, conducen a plantearnos la necesidad de cambio en la metodología de enseñanza por una que permita superar el esquema de enseñanza-aprendizaje que sobrevalora la habilidad memorística (Arriba Bueno \& García Sanchís, 2014). En ese sentido, la colaboración es una estructura de interacción diseñada para facilitar la obtención de un determinado producto final o meta a través de las personas que trabajan juntas en grupos Panitz (1997).

Los proyectos colaborativos promueven un nuevo modelo de aprendizaje basado en la realización de diferentes tareas, cuya ejecución requiere de la participación y colaboración de todos los componentes de un determinado equipo de trabajo. Así, a través de estos proyectos es posible llevar a cabo la construcción colectiva del conocimiento. Del Moral y Villalustre, (2007), un conocimiento intercambiable, acumulativo, colaborativo, que puede ser compartido, transferido y convertido en un bien público. Cobo y Pardo, (2007). A través de ello, se exige al estudiante que desempeñe un rol más activo, dinámico y comprometido con su propio aprendizaje, y solicitando al docente la creación de ambientes de aprendizaje que promuevan la interacción y comunicación entre los docentes, apoyándose en la utilización de herramientas tecnológicas para el desarrollo de actividades didácticas capaces de propiciar un proceso de construcción activo del conocimiento, así como la adquisición de nuevos aprendizajes por parte de los estudiantes a partir de la interacción mutua entre sí y con el propio contexto (Del Moral \& Villalustre, 2007; Muñoz Morejón, \& Vidal Ledo, 2016).

La wiki, herramienta de colaboración on-line, es uno de los muchos componentes Web 2.0 que se pueden utilizar para mejorar el proceso de aprendizaje. La wiki permite generar y publicar contenido facilmente, el cual puede ser actualizado on-line por cualquier miembro de una comunidad de aprendizaje, en cualquier momento, desde cualquier lugar con acceso a Internet, donde todos se encargarían de la vigilancia y control del contenido publicado, favoreciendo de esta forma la autoría del grupo (González, Flores, Orozco, 2008; García \& Gonzalez, 2017).

Las similitudes entre la teoría pedagógica del constructivismo y el nuevo marco del aprendizaje colaborativo que introduce la wiki, la convierten en herramienta idónea para la enseñanza on-line (Del Moral et al., 2007). Dada su naturaleza abierta y flexible, la wiki se convierte en una aplicación potenciadora del aprendizaje cooperativo y colaborativo que favorece el desarrollo de proyectos colaborativos gestionando el trabajo autónomo y grupal de los estudiantes, y pueden ser empleadas en la enseñanza universitaria, no sólo como recurso didáctico, sino también en la investigación y en la elaboración de diferentes proyectos docentes (Del Moral y Villalustre, 2007).

La wiki constituye una herramienta que permite fomentar la colaboración de los docentes y la participación de los alumnos en la construcción de su propio conocimiento a través de la generación y publicación de contenido en forma conjunta (Flores et al., 2008a). Por otro lado, según Boulos et al., (2006), la wiki activa la participación de los alumnos en la construcción de sus propios conocimientos, y pueden ser utilizadas en el aprendizaje para involucrar a los estudiantes con otras personas, dentro de entornos de colaboración (Parker \& Chao, 2007; Núñez, Míguez, \& Seoane, 2016; Vitalaru, B. 2019). 
Por tal motivo es importante conocer de cerca este fenómeno, la investigación tuvo como objetivo determinar en qué medida la utilización de la wiki permite mejorar la colaboración en el desarrollo de trabajos grupales en estudiantes universitarios de una universidad privada de Lima.

\section{Método}

El diseño metodológico de la investigación es cuasi-experimental ya que emplea un grupo de control y un grupo experimental. Durante el desarrollo de la investigación se organizaron dos grupos con estudiantes de asignaturas de enseñanza de algoritmo, grupo control (GC) y grupo experimental (GE), a ambos grupos se le aplicó un pretest (O1), luego se utilizó la wiki (X) sólo con el grupo experimental (GE) dentro de un aula virtual (contexto virtual) y, finalmente, a ambos grupos se aplicó un postest $(\mathrm{O} 2)$.

La población de estudiantes estuvo conformada por 280 estudiantes matriculados en las asignaturas de enseñanza de algoritmo de la Facultad de Ingeniería y Arquitectura (FIA) de la una universidad privada de Lima. Se empleó el muestreo de tipo probabilístico, ya que todos los elementos (alumnos) de la población tienen la misma probabilidad de salir elegidos en la muestra. El tamaño de la muestra ha sido calculado aplicando la fórmula de la Teoría Estadística del Muestreo obteniendo 80 estudiantes. El instrumento desarrollado tiene un total de 11 preguntas y se aplicó a los grupos de estudio después de la entrega del primer trabajo grupal (pretest) y después de la entrega del segundo trabajo grupal (postest). Para la calificación de los trabajos monográficos grupales de utilizó una rúbrica desarrollada por los docentes.

En el procesamiento de los datos se utilizó el programa estadístico SPSS en su versión 20.0. Se realizó un análisis estadístico descriptivo e inferencial, para verificar las hipótesis planteadas. La estadística descriptiva comprendió medidas de tendencia central y de variabilidad. En la prueba de hipótesis se utilizó la Prueba T para dos muestras independientes, que permitió contrastar hipótesis referidas a la diferencia entre dos medias independientes. Además, se aplicó la prueba de Levene de homogeneidad de varianzas, que consistió en un paso previo, a la prueba T para comparar dos medias de muestras independientes.

\section{Resultados}

En la tabla 1 se observa las medidas descriptivas de las puntuaciones correspondientes a las dimensiones del aprendizaje colaborativo, obtenidas en el pretest (antes de utilizar la wiki). Se puede ver que, en la interdependencia positiva, en la construcción de significados y en las relaciones psicosociales, los promedios de las puntuaciones obtenidos en el grupo experimental $(2.22,2.21$ y 2.19$)$ y en el grupo control $(2.08,2.03,2.09)$, se aproximan a 2 . Por lo que se puede ver que los promedios del grupo experimental se diferencian mínimamente de los promedios obtenidos en el grupo control, a pesar de que los valores en el grupo experimental son mayores a los del grupo control. Así también, se observa respecto al aprendizaje colaborativo, que los promedios obtenidos en ambos grupos, al igual que en sus dimensiones, no difieren mucho. En el grupo experimental es 2.20 y en el grupo control es 2.07 , valores que en ambos grupos se aproximan a 2 . Por otra parte, se puede apreciar respecto a la variabilidad de las puntuaciones, que los valores de las desviaciones estándar en el grupo experimental $(0.74,0.69,0.69)$, correspondientes a las tres dimensiones, fueron menores a los valores observados en el grupo control $(1.11,1.08,1.12)$.

Asimismo, en el aprendizaje colaborativo se observa que la desviación estándar en el grupo experimental (0.70) es menor al obtenido en el grupo control (1.10). 
Tabla 1.

Análisis descriptivo de los resultados antes del uso de la wiki

\begin{tabular}{|c|c|c|c|c|}
\hline & \multirow{2}{*}{ Medidas Descriptivas } & \multicolumn{2}{|c|}{ Pretest } \\
\hline & & & G. Experimental & G. Control \\
\hline \multicolumn{2}{|r|}{ Tamaño muestra (n) } & & 40 & 40 \\
\hline \multirow{4}{*}{$\begin{array}{c}\mathrm{D} \\
\mathrm{I} \\
\mathrm{M}\end{array}$} & \multirow{4}{*}{$\begin{array}{l}\text { I. Interdependencia } \\
\text { positiva }\end{array}$} & Media & 2.22 & 2.08 \\
\hline & & Desviación estándar & 0.74 & 1.11 \\
\hline & & Mínimo & 1 & 0 \\
\hline & & Máximo & 3.48 & 3.96 \\
\hline $\begin{array}{l}\mathrm{E} \\
\mathrm{N}\end{array}$ & \multirow{4}{*}{$\begin{array}{l}\text { II. Construcción de } \\
\text { significados }\end{array}$} & Media & 2.21 & 2.03 \\
\hline $\begin{array}{l}\mathrm{N} \\
\mathrm{S}\end{array}$ & & Desviación estándar & 0.69 & 1.08 \\
\hline $\begin{array}{l}\mathrm{S} \\
\mathrm{I}\end{array}$ & & Mínimo & 1.07 & 0 \\
\hline O & & Máximo & 3.37 & 3.83 \\
\hline $\mathrm{N}$ & \multirow{4}{*}{$\begin{array}{l}\text { III. Relaciones } \\
\text { psicosociales }\end{array}$} & Media & 2.19 & 2.09 \\
\hline $\mathrm{E}$ & & Desviación estándar & 0.69 & 1.12 \\
\hline $\mathrm{S}$ & & Mínimo & 1.12 & 0 \\
\hline & & Máximo & 3.4 & 3.96 \\
\hline \multirow{4}{*}{\multicolumn{2}{|c|}{ Aprendizaje colaborativo }} & Media & 2.20 & 2.07 \\
\hline & & Desviación estándar & 0.70 & 1.10 \\
\hline & & Mínimo & 1.1 & 0 \\
\hline & & Máximo & 3.38 & 3.88 \\
\hline
\end{tabular}

En la tabla 2, se observa las medidas descriptivas de las puntuaciones correspondientes a las dimensiones del aprendizaje colaborativo, obtenidas en el postest (después de utilizar la wiki). Se puede ver que en la interdependencia positiva, en la construcción de significados y en las relaciones psicosociales, los promedios de las puntuaciones obtenidos en el grupo experimental $(2.77,2.65 \mathrm{y}$ 2.69) son mayores a los promedios obtenidos en el grupo control $(2.15,2.12,2.11)$. Asimismo, los promedios del grupo experimental se aproximan a 3, mientras que los promedios del grupo control se aproximan a 2. Por lo que es posible suponer que la diferencia entre los promedios de ambos grupos, en cada dimensión, no es mínima. Respecto al aprendizaje colaborativo, se observa que el promedio obtenido en el grupo experimental (2.70) es mayor al promedio del grupo control (2.13). También es posible suponer que esta diferencia no es mínima, ya que el promedio en el grupo experimental se aproxima a 3 y en el grupo control se aproxima a 2 . Por otra parte, se puede ver respecto a la variabilidad de las puntuaciones, que los valores de las desviaciones estándar en el grupo experimental $(0.50,0.42,0.44)$, correspondientes a las tres dimensiones, fueron menores a los valores observados en el grupo control $(0.84,0.84,0.85)$. Asimismo, en el aprendizaje colaborativo se observa que la desviación estándar en el grupo experimental (0.44) es menor al obtenido en el grupo control (0.84). 
Tabla 2.

Análisis descriptivo de los resultados después del uso de la wiki

\begin{tabular}{|c|c|c|c|c|}
\hline & & & & \\
\hline & & Medidas Descriptivas & G. Experimental & G. Control \\
\hline & Tamaño muestra (n) & & 40 & 40 \\
\hline & & Media & 2.77 & 2.15 \\
\hline $\mathrm{D}$ & I. Interdependencia & Desviación estándar & 0.50 & 0.84 \\
\hline I & positiva & Mínimo & 1.6 & 0.2 \\
\hline M & & Máximo & 3.56 & 3.8 \\
\hline E & & Media & 2.65 & 2.12 \\
\hline $\mathrm{N}$ & II. Construcción de & Desviación estándar & 0.42 & 0.84 \\
\hline S & significados & Mínimo & 1.43 & 0.2 \\
\hline $\begin{array}{l}1 \\
0\end{array}$ & & Máximo & 3.33 & 3.8 \\
\hline $\mathrm{N}$ & & Media & 2.69 & 2.11 \\
\hline E & III. Relaciones & Desviación estándar & 0.44 & 0.85 \\
\hline $\mathrm{S}$ & psicosociales & Mínimo & 1.28 & 0.2 \\
\hline & & Máximo & 3.4 & 3.8 \\
\hline & & Media & 2.70 & 2.13 \\
\hline & & Desviación estándar & 0.44 & 0.84 \\
\hline & Aprendizaje colaborativo & Mínimo & 1.44 & 0.2 \\
\hline & & Máximo & 3.37 & 3.8 \\
\hline
\end{tabular}

En la Tabla 3, se observa el nivel de desarrollo respecto al aprendizaje colaborativo para el desarrollo de trabajos grupales de los alumnos del grupo control y del grupo experimental, al inicio de la investigación (pretest). En el grupo experimental, el $25 \%$ de los alumnos se encuentra en el nivel alto, un $32.5 \%$ en el nivel medio y un $42.5 \%$ en el nivel bajo. Mientras que en el grupo control, un $30 \%$ de los alumnos se encuentra en el nivel alto, un $10 \%$ en el nivel medio y un $60 \%$ restante en el nivel bajo. Es posible ver que el mayor porcentaje de alumnos, en ambos grupos, se encuentra en el nivel bajo. Así también el porcentaje en el nivel medio es mayor en el grupo experimental y el porcentaje en el nivel alto es mayor en el grupo control. Por otra parte, después de utilizar la wiki, para el desarrollo de trabajos grupales, en el grupo experimental y de realizar el postest, se observó que: en el grupo experimental el 32.5\% de los alumnos se encuentran en el nivel alto, un $57.5 \%$ en el nivel medio y un $10 \%$ en el nivel bajo. Mientras que en el grupo control, el $17.5 \%$ se encuentra en el nivel alto, un $40 \%$ en el nivel medio y un $42.5 \%$ en el nivel bajo. Se puede apreciar que luego del postest, en ambos grupos hubo una disminución del número de alumnos en el nivel bajo, pero esta disminución fue mayor en el grupo experimental (10\%). Respecto al nivel medio, en ambos grupos hubo un incremento porcentual. Además, es posible ver que el grupo experimental sufrió un incremento porcentual en el nivel alto (de 25\% a 32.5\%), mientras que el grupo control sufrió una disminución porcentual en este nivel (de 30\% a 17.5\%), luego del pos test. Lo que reflejaría que los resultados en el grupo experimental fueron más satisfactorios que en el grupo control. 


\section{Tabla 3.}

Nivel de desarrollo respecto al aprendizaje colaborativo, para el desarrollo de trabajos grupales (pretest y postest)

\begin{tabular}{lllllllll}
\hline Nivel & \multicolumn{2}{l}{ PRE TEST } & \multicolumn{5}{l}{ POS TEST } \\
& \multicolumn{2}{l}{ G. Experim. } & \multicolumn{2}{l}{ G. Control } & \multicolumn{2}{l}{ G. Experim. } & \multicolumn{2}{c}{ G. Control } \\
\hline Alto & 10 & $25 \%$ & 12 & $30 \%$ & 15 & $37.5 \%$ & 7 & $17.5 \%$ \\
Medio & 14 & $35 \%$ & 6 & $15 \%$ & 21 & $52.5 \%$ & 16 & $40 \%$ \\
Bajo & 16 & $40 \%$ & 22 & $55 \%$ & 4 & $10 \%$ & 17 & $42.5 \%$ \\
Total & 40 & $100 \%$ & 40 & $100 \%$ & 40 & $100 \%$ & 40 & $100 \%$ \\
\hline
\end{tabular}

Alto (15-20), Medio (11-14) у Bajo (0 - 10).

En la prueba de hipótesis sobre igualdad de varianzas (prueba de Levene), se observa que el valor del estadístico de contraste es $\mathrm{F}=14.679$ y el nivel crítico (Sig.) es $p=0$ el cual es menor a $\alpha=0.05$, entonces se rechaza la hipótesis de que las varianzas poblacionales son iguales. En la prueba T para igualdad de medias de SPSS, se observa que el estadístico $t=3.831$ con 59.014 g.l. (grados de libertad) y que el nivel crítico (Sig. bilateral) es $p=0$. Al encontrarnos ante un contraste unilateral del tipo "igual frente a mayor", debemos comparar "el área bajo la curva a la derecha del estadístico" con el valor $\alpha=0.05$. Es decir si $(p / 2)=0<0.05$ entonces se rechaza la hipótesis nula. Por tanto, se acepta que el promedio obtenido en el grupo experimental, correspondiente a las puntuaciones del aprendizaje colaborativo, es mayor al promedio del grupo control, después de utilizar la wiki en el grupo experimental, para la realización de trabajos monográficos grupales (Tabla 4).

\section{Tabla 4.}

Prueba para comparar las medias de los grupos control y experimental, respecto al aprendizaje colaborativo para el desarrollo de trabajos monográficos grupales

\begin{tabular}{lcc|ccc}
\hline & \multicolumn{2}{c|}{$\begin{array}{c}\text { Prueba de Levene para la } \\
\text { igualdad de varianzas }\end{array}$} & \multicolumn{3}{c}{$\begin{array}{c}\text { Prueba T para igualdad de } \\
\text { medias }\end{array}$} \\
\cline { 2 - 6 } & $\mathrm{F}$ & Sig. & t & g.l. & $\begin{array}{c}\text { Sig. } \\
\text { (bilateral) }\end{array}$ \\
\hline $\begin{array}{l}\text { Asumiendo varianzas } \\
\text { iguales }\end{array}$ & 14.679 & 0 & 3.831 & 78 & 0 \\
\hline $\begin{array}{l}\text { No asumiendo varianzas } \\
\text { iguales }\end{array}$ & & & & & \\
\hline
\end{tabular}

\section{Discusión}

El propósito del estudio fue identificar el uso de la wiki como herramienta de trabajo colaborativo en el desarrollo de trabajos grupales. La investigación encuentra que el uso de la wiki mejoró la construcción de significado y las relaciones psicosociales de los estudiantes universitarios demostrando que el aprendizaje colaborativo mejora con apoyo de la herramienta web, wiki. Resultados similares fueron reportados por Pérez, Paule, Gayo, Labra, Redondo, Fernández y Prado (2007) quienes describen la experiencia de la utilización de la wiki como herramienta facilitadora del planteamiento, resolución, recopilación, revisión y mejora de ejercicios relacionados 
con la algorítmica, en una asignatura de Ingeniería. Por otro lado, Arriba Bueno y García Sanchís (2014) indican que la wiki facilita el proceso de enseñanza-aprendizaje colaborativo, a partir de la asunción de un rol más activo del alumno y la monitorización por parte del profesor, la utilización de estas plataformas mejora las competencias analíticas de los alumnos y su capacidad de valoración crítica. Asimismo, Sánchez, Botella, del Mar y Brel, (2018) mencionan que las wikis han contribuido a mejorar aptitudes y habilidades de trabajo autónomo y en equipo, la planificación óptima de tiempos y espacios.

El avance de la tecnología digital ha creado entornos que favorecen en gran medida la interacción social. Un gran número de aplicaciones han sido diseñadas e implementadas para soportar el aprendizaje colaborativo, conectando los estudiantes, docentes y comunidad, de manera sincrónica o asincrónicamente, para realizar actividades colaborativas (Avello Martínez, \& Duart, 2016). Por su parte Herrada y Baños (2018) a partir de una revisión sistemática concluye que existe un creciente interés por parte de docentes e investigadores a la hora de implementar el aprendizaje cooperativo haciendo uso de plataformas virtuales de enseñanza-aprendizaje, a la vez que se observa un elevado nivel de satisfacción por parte de docentes y discentes involucrados en dichas experiencias.

Esta investigación nos deja una gran experiencia, cuyos resultados permiten sugerir que los docentes hagan uso de las wikis en las aulas, con el propósito de incrementar y/o fomentar la participación de los estudiantes, esto permite tener a los docentes actualizados en cuanto al uso de las TIC y las ventajas que éstas ofrecen en el aprendizaje colaborativo, con el propósito de promover aprendizajes significativos en los estudiantes.

\section{Referencias}

Abramson, L. (2011). Can social networking keep students inschool? NPR: Morning Edition. Recuperado de http://www.npr.org/2011/02/09/133598049/can-social-networking-keepstudents-in-school

Adell, J. (1997). Redes y educación. En De Pablos, J. y Jiménez, J. (Eds.). Nuevas tecnologías, comunicación audiovisual y educación. Barcelona: Cedecs.

Adell, J. (2006). Tendencias en educación en la sociedad de las tecnologías de la información. Edutec. Revista Electrónica de Tecnología Educativa, 7, a007. Doi: https://doi.org/10.21556/edutec.1997.7.570

Arriba Bueno, R., \& García Sanchís, M. C. (2014). La eficacia de la wiki como herramienta de aprendizaje colaborativo para aprender Política Económica Internacional. Digital Education Review, 26, 24-38. Doi: https://doi.org/10.1344/der.2014.0.24-38

Avello Martínez, R., \& Duart, J. M. (2016). New collaborative learning trends in e-learning: Keys for it effective implementation. Estudios pedagógicos (Valdivia), 42(1), 271-282. Doi: https://dx.doi.org/10.4067/S0718-07052016000100017

Boulos, M. N. K., Maramba, I., \& Wheeler, S. (2006). Wikis, blogs and podcasts: a new generation of Web-based tools for virtual collaborative clinical practice and education. BMC medical education, 6(1), 41. Recuperado de http://www.biomedcentral.com/content/pdf/1472-69206-41.pdf

Boza Carreño, A., Conde Vélez, S. (2015). Web 2.0 en educación superior : formación, actitud, uso, impacto, dificultades y herramientas. Digital Education Review, 28, 45-58. Doi: https://doi.org/10.1344/der.2015.28.45-58

Casanova, M. O., Alvarez, I. M., Alemany, I. (2009). Propuesta de indicadores para evaluar y promover el aprendizaje cooperativo en un debate virtual. EDUTEC, Revista Electrónica de Tecnología Educativa, 28. doi: http://dx.doi.org/10.21556/edutec.2009.28.455

Cobo, C., \& Pardo, H. (2007). Planeta Web 2.0. Inteligencia colectiva o medios fast food. 
Barcelona: México: Grup de Recerca d'Interaccions Digitals, Universitat de Vic. Flacso México.

Córdoba Castrillón, M. M., López Murillo, E. E., Ospina Moreno, J., \& Polo, J. A. (2017). Estudiantes de la básica y media con respecto al uso de las TIC como herramientas de apoyo a su aprendizaje. Trilogía Ciencia Tecnología Sociedad, 9(16), 113-125. doi https://doi.org/10.22430/21457778.178

Del Moral, M. E., \& Villalustre, L. (2007). Las wikis: construcción compartida del conocimiento y desarrollo de competencias. IV Jornadas Internacionales de Innovación Universitaria. Universidad Europea de Madrid. Madrid.

Flores, J., Del Moral, M. E. \& González, F. (2008). Propuesta de modelo wiki teórico para la enseñanza superior. V Congreso Iberoamericano de Docencia Universitaria (CIDU). Universidad Politécnica de Valencia. Valencia, España.

Gairin, J., \& Martin, M. (2004). Las instituciones educativas en la encrucijada de los nuevos tiempos: retos, necesidades, principios y actuaciones. Tendencias pedagógicas, 9, 21-44. Recuperado de https://revistas.uam.es/tendenciaspedagogicas/article/view/1839

García, J.., \& González, M. (2017). Entornos personales de aprendizaje de estudiantes universitarios costarricenses de educación: análisis de las herramientas de búsqueda de información. Revista de Investigación Educativa, 35(2), 389-407. doi: https://doi.org/10.6018/rie.35.2.253101

Gómez Francisco, T., \& Rubio González, J. (2017). Complejidad-educación: un aporte para las generaciones futuras. Educación y Humanismo, 19(33), 409-424. doi: https://doi.org/10.17081/eduhum.19.33.2653

González, F., Flores, J., Orozco, J. (2008). Experiencia en el uso de herramientas wiki para el aprendizaje. En: V Congreso Iberoamericano de Docencia Universitaria (CIDU). Universidad Politécnica de Valencia. Valencia, España.

Hernandez, R. M. (2017). Impacto de las TIC en la educación: Retos y Perspectivas. Propósitos y Representaciones, 5(1), 325-347. Doi: http://dx.doi.org/10.20511/pyr2017.v5n1.149

Herrada, H., \& Baños, R. (2018). Aprendizaje cooperativo a través de las nuevas tecnologías: Una revisión. @ tic revista d'innovació educativa, 20, 16-25. doi: https://doi.org/10.7203/attic.20.11266

Lozano Martínez, J., Ballesta Pagán, F. J., Alcaraz García, S., \& Cerezo Maíquez, M. (2013). Las Tecnologías de la Información y la Comunicación (TIC) en el proceso de enseñanza y aprendizaje del alumnado con trastorno del espectro autista (TEA). 14. Recuperado de https://revistascientificas.us.es/index.php/fuentes/article/view/2359

Marqués, P. (2013). Impacto de las TIC en la educación: funciones y limitaciones. 3C TIC, 2(1). Recuperado de https://www.3ciencias.com/articulos/articulo/impacto-de-las-tic-en-laeducacion-funciones-y-limitaciones/

Muñoz Morejón, M., \& Vidal Ledo, M. (2016). Plataformas de trabajo colaborativo para la dirección en Salud, aprender desde la educación virtual. Revista de información científica para la dirección en salud. INFODIR, O(23), 66-76. Recuperado de http://www.revinfodir.sld.cu/index.php/infodir/article/view/187

Núñez, I., Míguez, M., \& Seoane, G. (2016). Wikis en Moodle: la mirada de estudiantes y docentes. Educación química, 27(4), 257-263. Doi: https://doi.org/10.1016/j.eq.2016.08.001

Panitz, T. (1997). Collaborative versus Cooperative Learning: Comparing the two definitions helps understand the nature of interactive learning. Cooperative Learning and College Teaching, 8(2). Recuperado de http://home.capecod.net/ tpanitz/tedsarticles/coopdefinition.htm

Parker, K., \& Chao, J. (2007). Wiki as a teaching tool. Interdisciplinary Journal of e-learning and Learning Objects, 3(1), 57-72. Recuperado de http://www.ijklo.org/Volume3/IJKLOv3p057-072Parker284.pdf

Pérez, J., Paule, Mà., Gayo, D., Labra, J., Redondo, J., Fernández, C. \& Prado, M. (2007); Empleo de wikis como apoyo en desarrollo colaborativo de ejercicios. En: II Jornadas de 
Intercambio de Experiencias en Docencia Universitaria. Universidad de Oviedo, Oviedo.

Rodrigo Cano, D., Aguaded Gómez, J.I., García Moro, F.J. (2019). Metodologías colaborativas en la Web 2.0: el reto educativo de la Universidad. REDU. Revista de Docencia Universitaria, 17(1), 229-244. doi: https://doi.org/10.4995/redu.2019.10829

Sánchez, E. M. S., Rodríguez, E. B., Villar, M. C., \& Cachón, M. P. B. (2018). Las TIC y las prácticas de historia económica: elaboración de wikis colaborativas en el campus virtual. In Investigaciones en historia económica: su transferencia a la docencia (pp. 401-413). Departament d'Economia Aplicada.

Vitalaru, B. (2019). Wikis como herramientas de trabajo colaborativo complementario y desarrollo de recursos para la formación en traducción a nivel universitario: Análisis retrospectivo. Revista de Educación a Distancia, 1(59), 1-47. doi: https://doi.org/10.6018/red/59/02 